\title{
INFLUENCIA PERSONAL DE LOS CANDIDATOS Y COMPETENCIA ESPACIAL EN LAS ELECCIONES PRESIDENCIALES BRASILEÑAS ENTRE 2006 y 2014
}

\author{
Personal influence of candidates and spatial competition in the \\ brazilians elections between 2006-2014
}

Luis Eduardo LEÓN GANATIOS

Julen BERASALUCE ${ }^{2}$

\section{Sumario:}

Introducción. 2. El sistema de partidos en Brasil. 3. El peso de la ideología en el sistema de partidos en Brasil. 4. La variable personalista en la competencia espacial. 5. Análisis de la variable personalista en las elecciones presidenciales brasileñas de 2006,2010 y 2014. 6. Conclusiones. Referencias.

Resumen: La medición de la influencia relativa de los candidatos electorales puede estar sujeta a cuestiones ideológicas. Justificando adecuadamente la consideración de un modelo de competencia espacial con dos partidos para el caso de Brasil, comparamos los resultados de las elecciones presidenciales con el esperado, por la distribución ideológica de los brasileños. A partir de esta comparación ofrecemos una clasificación sobre la valencia o calidad de los candidatos para las elecciones presidenciales en el período de 2006 a 2014.

Palabras Clave: Competencia espacial. Elecciones. Candidatos. Valencia. Sistema de Partidos. Brasil.

Abstract: The measurement of the relative influence of electoral candidates could be biased by ideology. We properly justify considering a model of spatial competition with two parties for Brazil. We compare the results of the presidential elections with the expected ones from the ideological distribution of Brazilians. Through this comparison we provide a ranking of the valence or quality of the candidates for the presidential elections of 2006 to 2014.

Key words: Spatial Competition. Elections. Candidates. Valence. Party Systems. Brazil.

\section{INTRODUCCIÓN}

En cada elección pueden valorarse las capacidades personales de cada candidato. Al término de la misma, la definición de ganadores y perdedores ofrece un escenario final sobre las

\footnotetext{
${ }^{1}$ Profesor de Carrera de tiempo completo de División de Estudios Políticos de la Universidad de Guanajuato, ganatios.leon@gmail.com

${ }^{2}$ Licenciado en economía por la Universidad del País Vasco (España). Maestro por la Universidad de Barcelona, España. Doctor en International Doctoráte in economic analysis por la Universidad Autónoma de Barcelona. Profesor Titula del Colegio de México. Miembro del sistema Nacional de Investigadores.
} 
supuestas habilidades e influencias de los participantes. No obstante, el resultado final no depende solo de las capacidades personales de un candidato, sino también de la posición ideológica que representa. Por ello, proponemos un modelo y su aplicación a las tres últimas elecciones presidenciales en Brasil, con el fin de conocer cuál es la calidad y la influencia relativa de los principales participantes.

Para ello, definiremos el tipo de competencia partidista existente en Brasil, con el fin de justificar adecuadamente los supuestos del modelo; en concreto, el número de partidos, la polarización ideológica, la importancia de las características personales y la consideración de competencia espacial. La competencia espacial presupone la existencia de un espacio ideológico que los partidos políticos emplean para situarse y según el cual los ciudadanos emiten su voto. Con base en este modelo, observaremos los resultados esperados e introduciremos un término de calidad en los candidatos que obtendremos endógenamente, exigiendo que el mismo refleje el resultado electoral.

La toma en consideración del modelo de competencia espacial implica la suposición de una forma de utilidad concreta de los ciudadanos con respecto al voto. Entre las mayores suposiciones que tenemos que realizar en este modelo figura la consideración de que todos los votantes, si bien pueden tener ideales distintos, tienen utilidades simétricas con respecto a su ideal. Además hemos de suponer también que las funciones de utilidad son homogéneas. Sin embargo, los resultados obtenidos no dependen de las subjetividades ideológicas a la hora de emitir un juicio.

A pesar de lo reductivo de los argumentos, consideramos que la participación de dos partidos principales en las elecciones presidenciales brasileñas de los últimos años brinda un escenario particular para la aplicación de esta técnica. La tendencia de apoyo de muchos grupos políticos a un candidato u otro manifiesta a priori la importancia personal de los candidatos participantes puesto que ofrece suficientes posibilidades analíticas para contemplar las repercusiones del modelo.

\section{EL SISTEMA DE PARTIDOS EN BRASIL}

A partir de 1988 la elección de presidente en Brasil es directa; El candidato necesita mayoría absoluta. De no alcanzar ningún candidato esta mayoría habrá una segunda vuelta con los candidatos más votados y renuevan cada cuatro años. ${ }^{3}$

Con respecto a la ideología el sistema de partidos cubre totalmente el continuo izquierda - derecha, teniendo a partidos como el Partido de los Trabajadores (PT), en el centro con el Partido de la Socialdemocracia Brasileña (PSDB) y el Partido del Frente Liberal (PFL) como ejemplos, quienes se correlacionan con las divisiones ideológicas. ${ }^{4}$

Uno de los indicadores propuestos por Sartori ${ }^{5}$ para definir la evolución y estabilidad del sistema de partidos es el número efectivo de partidos, ${ }^{6}$ indice que permite apreciar cuántos

\footnotetext{
${ }^{3}$ NOHLEN, D., Sistemas electorales y partidos políticos, México, Fondo de Cultura Económica, 2004.

${ }^{4}$ ALCANTARA, M., Sistemas políticos de América Latina, Volumen I: América del Sur, Madrid, Editorial TECNOS, 1999.

${ }^{5}$ SARTORI, G., Partidos y sistemas de partido, Madrid: Alianza Editorial, 1987.

${ }^{6}$ El índice de número efectivo de partidos de Laakso y Taagepera se calcula con:
}

$$
N=1 / \sum_{i=1}^{n} p_{i}^{2}
$$

donde $\mathrm{p}_{\mathrm{I}}^{2}$ es el resultado relativo obtenido por cada partido para el total de partidos, p. 36., 1999. 
partidos compiten electoralmente, y toma en cuenta el número de partidos reales que consiguen al menos un $10 \%$ de los votos.

Tabla 1. Número efectivo de partidos en Brasil entre las elecciones de 1989-2014

\begin{tabular}{|c|c|c|c|c|c|c|c|}
\hline Año & 1989 & 1994 & 1998 & 2002 & 2006 & 2010 & 2014 \\
\hline $\begin{array}{c}\text { Número efectivo } \\
\text { de partidos }\end{array}$ & 7.69 & 2.77 & 2.56 & 3.33 & 2.5 & 3.03 & 2.94 \\
\hline
\end{tabular}

Elaboración propia a partir de resultados electorales. ${ }^{8}$

Se puede observar en la anterior tabla que sólo se observa un alto número efectivo de partidos en las elecciones de 1989, a partir de allí se mantienen dos tendencias, un multipartidismo limitado y un bipartidismo, tal como señala Meneguello se confirma un clivaje desde 1994, que consiste en un contorno bipartidista en un escenario de múltiples partidos, el PT y PSDB generan una polarización nítida. ${ }^{9}$

El sistema partidista brasileño es altamente personalista, a juicio de esta investigación, tal como señala Nohlen Es válido destacar la impresionante orientación del voto hacia los candidatos, el elector vota por personas y no por listas, esto es, un indicador fuerte del deficiente arraigo de los partidos políticos. ${ }^{10}$

Tabla 2. Resultados electorales por candidatos en Brasil entre 1989 y 2014

\begin{tabular}{|c|c|c|c|c|c|c|c|}
\hline Candidato & 1989 & 1994 & 1998 & 2002 & 2006 & 2010 & 2014 \\
\hline Collor de Melo & $\mathbf{2 8 . 5 2 / 5 3 . 0 3}$ & $\mathrm{X}$ & $\mathrm{X}$ & $\mathrm{X}$ & $\mathrm{X}$ & $\mathrm{X}$ & $\mathrm{X}$ \\
\hline Lula da Silva & $16.08 / 46.97$ & 27.04 & 31.71 & $\mathbf{4 6 . 4 0 / 6 1 . 3}$ & $\mathbf{4 6 . 6 1 / 6 0 . 8}$ & $\mathrm{X}$ & $\mathrm{X}$ \\
\hline $\begin{array}{c}\text { Fernando } \\
\text { Cardoso }\end{array}$ & $\mathrm{X}$ & $\mathbf{5 4 . 2 7}$ & $\mathbf{5 3 . 0 6}$ & $\mathrm{X}$ & $\mathrm{X}$ & $\mathrm{X}$ & $\mathrm{X}$ \\
\hline José Serra & $\mathrm{X}$ & $\mathrm{X}$ & $\mathrm{X}$ & $23.20 / 38.7$ & $\mathrm{X}$ & $32.61 / 43.95$ & $\mathrm{X}$ \\
\hline Gerardo Alckmin & $\mathrm{X}$ & $\mathrm{X}$ & $\mathrm{X}$ & $\mathrm{X}$ & $41.63 / 39.2$ & $\mathrm{X}$ & $\mathrm{X}$ \\
\hline Dilma Rousseff & $\mathrm{X}$ & $\mathrm{X}$ & $\mathrm{X}$ & $\mathrm{X}$ & $\mathrm{X}$ & $\mathbf{4 6 . 9 1 / 5 6 . 0 5}$ & $\mathbf{4 1 . 5 9 / 5 1 . 6 4}$ \\
\hline Aécio Neves & $\mathrm{X}$ & $\mathrm{X}$ & $\mathrm{X}$ & $\mathrm{X}$ & $\mathrm{X}$ & $\mathrm{X}$ & $33.55 / 48.36$ \\
\hline
\end{tabular}

Elaboración propia con base en resultados electorales de database of the americas.

Se puede observar en la anterior tabla que en un total de 7 elecciones hay sólo 7 candidatos relevantes, que obtuvieron bien el primer lugar o el segundo en las elecciones efectuadas en este periodo, de estos 7 candidatos, Collor de Melo pertenecía al Partido de la Reconstrucción Nacional (PRN, actual Partido Laborista Cristiano) de resto los demás candidatos

\footnotetext{
7 OÑATE, P. \& OCAÑA, F., Análisis de datos electorales, Madrid, Centro de Investigaciones Sociológicas, 1999.

${ }^{8}$ El número efectivo de partidos ha sido calculado en base a los resultados de la primera vuelta. Los resultados electorales han sido obtenidos de Political Database of the Americas a excepción de los resultados de 2014 que fueron obtenidos del Tribunal Supremo Electoral de Brasil.

${ }^{9}$ MENEGUELLO, R., Las elecciones brasileñas de 2010: política nacional, fragmentación partidista y coaliciones. En Alcántara y Tagina (coord.) Elecciones y política en América Latina. México, Editorial Porrúa., 2013.

${ }^{10}$ NOHLEN, D., Op. Cit., nota 3, p. 358.
} 
pertenecen a dos partidos; Lula da Silva y Dilma Rousseff pertenecen al PT y Cardoso, Serra, Alckmin y Neves pertenecen al PSDB.

Esto muestra la existencia de un claro bipartidismo, con respecto al número efectivo de partidos.

\section{EL PESO DE LA IDEOLOGÍA EN EL SISTEMA DE PARTIDOS EN BRASIL}

Con el fin de aplicar un modelo de competencia espacial en las elecciones presidenciales desde 2006 hasta $2014^{11}$ en Brasil, resulta importante definir los criterios ideológicos que puedan permitir analizar dicha competencia electoral en términos de una escala lineal de valores y la posterior ubicación de partidos políticos y votantes en la misma.

Sartori estableció un índice de polarización que ayudaba a establecer el peso y distancia ideológicos dentro de una elección, entendiendo dicha polarización como el ámbito general del espectro ideológico de cualquier comunidad política dada ${ }^{12}$ (Sartori, 1987, p. 161). Si se habla de polarización de un sistema de partidos debe referirse al modelo espacial propuesto originariamente por Downs. ${ }^{13}$

Tabla 3. Ubicación ideológica de los partidos políticos brasileños

\begin{tabular}{|c|c|c|c|}
\hline Partido & $\mathbf{2 0 0 6}$ & $\mathbf{2 0 1 0}$ & $\mathbf{2 0 1 4}$ \\
\hline PT & 4.43 & 4.61 & 5.35 \\
\hline PSDB & 6.89 & 6.18 & 5.94 \\
\hline
\end{tabular}

Elaboración propia basada en la recopilación y construcción de las ubicaciones ideológicas de los partidos políticos.

Se puede observar que entre 2006 y 2014 la distancia entre los partidos ha disminuido y se han acercado al centro, lo que supone una competencia centrípeta entre los partidos políticos que configuran el bipartidismo brasileño de los últimos diez años.

Tabla 4.Polarización ponderada y polarización ponderada escalar en las elecciones presidenciales entre 2006 y 2014

\begin{tabular}{|c|c|c|c|}
\hline Polarizaciones & $\mathbf{2 0 0 6}$ & $\mathbf{2 0 1 0}$ & $\mathbf{2 0 1 4}$ \\
\hline Polarización Ponderada & 1.51 & 1.23 & 0.08 \\
\hline Polarización Ponderada Escalar & 2.41 & 2.21 & 0.56 \\
\hline
\end{tabular}

Elaboración propia a partir de la fórmula de polarización ponderada y polarización ponderada escalar

En la anterior tabla se calcula el índice de polarización ponderada que difiere al índice clásico propuesto por Sartori, en el sentido de introducir un mecanismo que pondere adecua-

\footnotetext{
${ }^{11}$ Sólo se realizará el análisis de las tres últimas elecciones, entre 2006 y 2014 debido a la dificultad de obtención de datos en elecciones anteriores para viabilizar el modelo aplicado.

${ }^{12}$ SARTORI, G., Partidos y sistemas de partido, Op. Cit., nota 5, p. 161, 1987.

${ }^{13}$ DOWNS, A., An economic theory of democracy. Nueva York, Harper y Row, 1957. 
damente la importancia que tiene cada partido con respecto a sus resultados electorales. ${ }^{14}$

Se puede observar que la polarización, que en ningún periodo es alta, va disminuyendo elección tras elección, comenzando con un índice de 1.51 en el 2006 para ubicarse en apenas un 0.08 en las elecciones de 2014.

La polarización reitera la tendencia centrípeta de los partidos políticos brasileños, En general, los sistemas bipartidistas15 se explican conforme a los criterios formalizados por el modelo de Downs de la competencia partidista. El modelo predice que en un sistema bipartidista los partidos competirán de forma centrípeta, moderando las divisiones y jugando la partida política con una moderación responsable. ${ }^{16}$

El análisis y revisión de las variables, número efectivo de partidos y polarización ponderada que determinan la existencia de un bipartidismo hace que la inclusión de la valencia se prevea de especial relevancia, dada la competencia centrípeta (en torno al centro), puesto que en ella la diferenciación ideológica entre partidos es menor.

Por tanto, en principio, la inclusión de la variable personalista o de valencia debería explicar la intención de voto de los electores brasileños entre las elecciones de 2006 y 2014.

\section{LA VARIABLE PERSONALISTA EN LA COMPETENCIA ESPACIAL}

La teoría espacial del voto, también conocida como el modelo clásico de proximidad, fue primeramente propuesta por Anthony Downs ${ }^{17}$ con base en un modelo de competencia espacial de Hotelling. ${ }^{18}$ Bajo el supuesto de dos partidos similares, motivados únicamente por la victoria electoral y que compitieran con la elección de un punto en el espacio ideológico unidimensional, el modelo prevé que los dos partidos ofrecerían una misma plataforma ideológica, idéntica a la preferida por el ciudadano mediano de la población.

Otro de los supuestos básicos del modelo lo componen las preferencias unimodales de los ciudadanos, moda constituida por su ideal político y ubicado en una escala unidimensional. En su aplicación práctica la escala más empleada comprende del o al 10, correspondiendo el primer valor a la izquierda extrema y el segundo a la extrema derecha. La referencia

${ }^{14}$ HAZAN, Reuven, Centre paties: Polarization and competition in european parlamentary democracies, Londres, Editorial Pinter, 1997. Hazan señala que el índice de Sartori se limita a medir la distancia entre los dos partidos más extremos, incurriendo en una exageración de la medida de la polarización, por tanto resulta más preciso utilizar el índice de polarización ponderada cuya fórmula es:

$$
P P=\sum_{i=1}^{n} \frac{v_{i}}{100}\left(x_{i}-\bar{x}\right)^{2}
$$

siendo vi el porcentaje de votos obtenidos en una elección; xi la posición que ocupa el partido político en la escala 1-10 y $\bar{x}$ la media de las posiciones de los partidos en la escala. La polarización ponderada escalar consiste en adecuar el resultado a una escala lineal de valores comprendida entre 1 hasta 10, y este se obtiene aplicando una raíz cuadrada y después multiplicando el resultado por dos.

15 A juicio de esta investigación consideramos que Brasil posee un sistema bipartidista a partir de 1994, aunque pueda considerarse un multipartidismo limitado o un sistema de alianzas y bloques entre partidos. El bipartidismo se mantiene aún así un solo partido (PT) haya triunfado en tres elecciones seguidas, pero en donde se cumplen las siguientes condiciones i) dos partidos se hallan en condiciones de competir por la mayoría absoluta de escaños, ii)uno de los dos partidos logra efectivamente conseguir una mayoría parlamentaria suficiente iii) este partido está dispuesto a gobernar solo y iv) la alternación o rotación en el poder sigue siendo una expectativa creíble.

${ }^{16}$ SARTORI, G., Op. Cit., nota 5, p. 249, 2009.

${ }^{17}$ DOWNS, A., Op. Cit., nota 13 .

${ }^{18}$ HOTELLING, H. Stability in competition, Economic Journal. 39: 41-57, 1929. 
izquierda-derecha, de usual aplicación en muchos países, puede tener un significado distinto en diferentes escenarios: conservadurismo-progresismo; mayor o menor intervención en los mercados; etc.

A fin de aplicar el modelo, hemos de suponer, además de las preferencias unimodales, una misma función de utilidad para todos los ciudadanos considerados, que además, sea simétrica,

$$
U\left(x_{i}, \pi_{j}, v_{j}\right)=-\left|x_{i}-\pi_{j}\right|+v_{j}
$$

donde $\mathrm{x}^{\mathrm{i}}$ expresa el ideal del ciudadano $\mathrm{i}$, mientras que $\pi \mathrm{j}$ se refiere a la plataforma del partido político j. El modelo se basa en el supuesto de que, en ausencia de costos de votación, el ciudadano i votará al partido que minimice la distancia de su plataforma con respecto al ideal mencionado.

Una de las variantes más consideradas sobre el modelo original es la inclusión de la variable de valencia, también conocida como variable personalista, primeramente planteada por Stokes ${ }^{19}$ como crítica al modelo espacial. El modelo espacial considera la heterogeneidad de los votantes, en función de sus distintos ideales, que comúnmente se clasifican en izquierdaderecha. Sin embargo, Stokes consideraba que algunas dimensiones como la disminución de la criminalidad o el crecimiento económico eran compartidas por todos los ciudadanos, por lo que el buen desempeño de un candidato en las mismas, habría de ser igualmente bien recibido por todos los ciudadanos.

A pesar de que la variable de valencia surgió como una crítica, ha sido finalmente incluida en los modelos de competencia espacial. Su inclusión en los modelos de competencia espacial, desarrollados por Ansolabhere \& Snyder ${ }^{20}$ así como por Aragonès \& Palfrey, ${ }^{21}$ ofrece resultados interesantes, como la no convergencia de candidatos en el mediano. Asimismo, la variable de valencia ha sido empleada en modelos de publicidad política, como el de Witt$\operatorname{man}^{22}$

${ }^{19}$ STOKES, D., Spatial models of party competition, American Political Science Review, Vol. 57, Núm. 2, 368$377,1963$.

${ }^{20}$ ANSOLABEHERE, S. \& SNYDER, J., Valence politics, Public Choice, Vol. 103, 327-226, 1999.

${ }^{21}$ ARAGONES, E. \& PALFREY, T., Mixed equilibrium in a Downsian Model with a Favored Candidate, Journal of Economic Theory. Vol. 103, 131-161, 2003.

${ }^{22}$ WITTMAN, D., Candidate quality, pressure group endorsements and the nature of political advertising, European Journal of Political Economy, Vol. 23, 360-378, 2007. 


\section{ANÁLISIS DE LA VARIABLE PERSONALISTA EN LAS ELECCIONES PRESIDENCIALES BRASILEÑAS DE 2006, 2010 Y 2014}

En nuestro caso consideraremos que los ciudadanos otorgarán su voto al candidato que les dé una mayor utilidad en función de la siguiente expresión:

$$
U\left(x_{i}, \pi_{j}\right)=-\left|x_{i}-\pi_{j}\right|
$$

donde VJ expresa la valencia o variable personalista del candidato del partido $\mathrm{j}$.

Consideremos la autoubicación ideológica de los ciudadanos en una escala de o a 10, en el que o representa la extrema izquierda y 10 la extrema derecha. A fin de evitar las discontinuidades que genera una escala con categorías discretas, redefinimos las categorías, convirtiéndolas en segmentos, de manera tal que el valor discreto original se ubique en el centro de la nueva categoría, a excepción de los dos valores extremos.

Tabla 5. Distribución ideológica en Brasil (2006)

\begin{tabular}{|c|c|c|c|c|c|}
\hline $\begin{array}{c}\text { Nivel } \\
\text { discreto }\end{array}$ & $\begin{array}{c}\text { Nivel } \\
\text { continuo }\end{array}$ & $\begin{array}{c}\text { Frecuencia } \\
\text { absoluta }\end{array}$ & $\begin{array}{c}\text { Frecuencia } \\
\text { relativa }\end{array}$ & Acumulado & $\begin{array}{c}\text { Acumulado } \\
\text { inverso }\end{array}$ \\
\hline 0 & $0-0.5$ & 34 & 0,0371 & 0,0371 & 1,0000 \\
\hline 1 & $0.5-1.5$ & 32 & 0,0349 & 0,0721 & 0,9629 \\
\hline 2 & $1.5-2.5$ & 61 & 0,0666 & 0,1386 & 0,9279 \\
\hline 3 & $2.5-3.5$ & 106 & 0,1157 & 0,2544 & 0,8614 \\
\hline 4 & $3.5-4.5$ & 104 & 0,1135 & 0,3679 & 0,7456 \\
\hline 5 & $4.5-5.5$ & 271 & 0,2959 & 0,6638 & 0,6321 \\
\hline 6 & $5.5-6.5$ & 56 & 0,0611 & 0,7249 & 0,3362 \\
\hline 7 & $6.5-7.5$ & 60 & 0,0655 & 0,7904 & 0,2751 \\
\hline 8 & $7.5-8.5$ & 60 & 0,0655 & 0,8559 & 0,2096 \\
\hline 9 & $8.5-9.5$ & 34 & 0,0371 & 0,8930 & 0,1441 \\
\hline 10 & $9.5-10.0$ & 98 & 0,1070 & 1,0000 & 0,1070 \\
\hline
\end{tabular}

Elaboración propia a partir de los datos de LAPOP.

En la distribución ideológica anterior no se han considerado las respuestas de las personas que no se ubicaban en dicha dimensión (122), las que no respondían (54) y las que no sabían (112), que junto con las 916 respuestas consideradas, conformarían el total de la muestra. Como es habitual en este tipo de distribuciones, la moda se sitúa en el valor central (5). Nos encontramos con que un mayor número de personas se ubican en el centro izquierda, puesto que un $36.79 \%$ de los que respondieron dieron un valor igual o inferior a 4 en contraposición al $33.62 \%$ que dio un valor mayor o inferior a 6. Esta ligera ventaja del sector ideológico de 
la izquierda no evita que la categoría central, con un $29.59 \%$ de los ciudadanos, contenga la clave para la victoria electoral en una competencia espacial.

Tabla 6. Resultados esperados y efectivos para las elecciones presidenciales en Brasil (2006)

\begin{tabular}{|c|c|c|c|c|}
\hline Partido & $\begin{array}{c}\text { Posición } \\
\text { partido }\end{array}$ & Plataforma partido & $\begin{array}{c}\text { Resultado esperado por } \\
\text { plataforma }\end{array}$ & Resultado final \\
\hline PT & 4.43 & $0-5.66$ & $67.35 \%$ & $60.83 \%$ \\
\hline PSDB & 6.89 & $5.66-10$ & $32.65 \%$ & $39.17 \%$ \\
\hline
\end{tabular}

Elaboración propia a partir de los datos de LAPOP.

Realizamos el análisis de los resultados de la segunda vuelta de las elecciones presidenciales de 2006, a la que llegaron como contendientes el entonces presidente Luis Inázio Lula da Silva por el PT y Gerardo Alckmin por el PSDB. La aplastante victoria de Lula da Silva, no habría de trasladarse automáticamente a una consideración de una mayor calidad de dicho candidato, por el papel de las posiciones ideológicas en el voto. De hecho, el PT era considerado por los votantes un partido mucho más centrista que el PSDB, con una diferencia tal que dicho partido, en función de las ubicaciones relativas, habría de capturar todo el espacio central y un $16 \%$ del primer segmento espacial del centro-derecha. En función de lo anterior, que sería el resultado esperable en caso de un voto ideológico con costos de alejamiento simétricos y para los datos considerados, el PT debería haber obtenido un $67.35 \%$ del total de los votos.

La diferencia de dicho porcentaje con el resultado real, podría haber sido explicado en términos de la diferencia de valencia entre los candidatos. Para replicar el resultado real, de un $60.83 \%$ de los votos para Lula da Silva, con la distribución ideológica considerada, la plataforma ideológica capturada por dicho contendiente habría tenido que ser el total del sector centro izquierda $(36.79 \%)$ y el $81.25 \%$ del segmento central, lo que implicaría la captura de la plataforma $[0,5.31)$. A fin de que se redujera la plataforma electoral del candidato Lula da Silva, dentro del modelo de competencia espacial con valencia, la valencia del candidato Gerardo Alckmin tendría que haber sido superior a la de Lula da Silva en el equivalente a o.6949 puntos del espacio ideológico considerado. Este resultado indica que la victoria de Lula da Silva, analizada desde un punto de vista de competencia espacial ideológica, provino de la posición más centrada del PT en comparación a la del PSDB, en vez de una ventaja personalista.

El resultado es consistente con la propia distribución de los votantes dentro de cada uno de los espectros ideológicos. Los votantes del sector centro-izquierda estaban más sesgados hacia el centro en comparación a los votantes de centro-derecha, en la que la cola extrema 
era más pesada. Ello podría haber influido en la selección de un candidato con menos opciones a disputar el centro político.

Tabla 7. Distribución ideológica en Brasil (2010)

\begin{tabular}{|c|c|c|c|c|c|}
\hline $\begin{array}{c}\text { Nivel dis- } \\
\text { creto }\end{array}$ & Nivel continuo & $\begin{array}{c}\text { Frecuencia } \\
\text { absoluta }\end{array}$ & $\begin{array}{c}\text { Frecuencia } \\
\text { relativa }\end{array}$ & Acumulado & $\begin{array}{c}\text { Acumulado } \\
\text { inverso }\end{array}$ \\
\hline 0 & $0-0.5$ & 97 & 0,0879 & 0,0879 & 1,0000 \\
\hline 1 & $0.5-1.5$ & 26 & 0,0236 & 0,1114 & 0,9121 \\
\hline 2 & $1.5-2.5$ & 33 & 0,0299 & 0,1413 & 0,8886 \\
\hline 3 & $2.5-3.5$ & 55 & 0,0498 & 0,1911 & 0,8587 \\
\hline 4 & $3.5-4.5$ & 42 & 0,0380 & 0,2292 & 0,8089 \\
\hline 5 & $4.5-5.5$ & 219 & 0,1984 & 0,4275 & 0,7708 \\
\hline 6 & $5.5-6.5$ & 63 & 0,0571 & 0,4846 & 0,5725 \\
\hline 7 & $6.5-7.5$ & 77 & 0,0697 & 0,5543 & 0,5154 \\
\hline 8 & $7.5-8.5$ & 128 & 0,1159 & 0,6703 & 0,4457 \\
\hline 9 & $8.5-9.5$ & 86 & 0,0779 & 0,7482 & 0,3297 \\
\hline 10 & $9.5-10.0$ & 278 & 0,2518 & 1,0000 & 0,2518 \\
\hline
\end{tabular}

Elaboración propia con datos de LAPOP.

La distribución ideológica de 2010 presenta algunas similitudes con la de 2006, aunque también cuenta con peculiaridades interesantes. A pesar de que el valor central continúa presentándose como una categoría importante, deja de ser la moda de la distribución, la cuál se ubica en 10, el valor de la extrema derecha. De hecho, el sector centro-derecha (categoría 6 y superiores) aglutinó el $57.25 \%$ de los ciudadanos, mientras que apenas el $\mathbf{2 2 . 9 2 \%}$ de la población se ubicaba en el sector centro-izquierda (categoría 4 e inferiores). Además de la traslación de la ciudadanía hacia la derecha ideológica, se observa una radicalización en las posturas, dada la mayor importancia relativa de los extremos. En este caso, el mediano de la población se sitúa en el 7, considerando valores discretos, o en el 6.7208 de nuestra transformación continua.

Tabla 8. Resultados esperados y efectivos para las elecciones presidenciales en Brasil (2010)

\begin{tabular}{|c|c|c|c|c|}
\hline Partido & Posición partido & Plataforma partido & $\begin{array}{c}\text { Resultado esperado por } \\
\text { plataforma }\end{array}$ & Resultado final \\
\hline PT & 4.61 & $0-5.395$ & $40.67 \%$ & $56.05 \%$ \\
\hline PSDB & 6.18 & $5.395-10$ & $59.33 \%$ & $43.95 \%$ \\
\hline
\end{tabular}

Elaboración propia con base en LAPOP.

La diferencia básica en la traslación ideológica de la población hacia la derecha genera una explicación totalmente distinta a lo ocurrido en la segunda vuelta de las elecciones presidenciales brasileñas de 2010. Dado el hecho de que los partidos contendientes fueron los 
mismos que en 2006, podemos apreciar que la posición de ambos partidos fue más centrada que la de 2006. En función de que este acercamiento fue mayor para el PSDB, se observa que la plataforma ideológica del PT se reduce al espacio [0, 5.395), bajo el supuesto de un comportamiento simétrico de los votantes, mientras que el PSDB obtendría el espacio restante. A pesar de que el espacio ideológico sería mayor para el PT, la mayor concentración ciudadana en el sector centro-derecha supondría una victoria esperada para el PSDB, por motivos ideológicos, con un $59.33 \%$ de los votos.

El resultado final obtenido por los candidatos Dilma Rousseff (PT) y José Serra (PSDB) en la segunda vuelta de las elecciones presidenciales de 2010 distó considerablemente de la estimación ofrecida en términos de voto ideológico. Dilma Rousseff obtuvo una victoria aplastante, con el 56.05\% de los votos, que, en función de la distribución ideológica del momento, tendría que ser explicado por una diferencia fundamental en la variable de valencia.

Para obtener el resultado final Dilma Rousseff habría tenido que capturar el segmento [o, 7,553), o lo que es lo mismo todas las categorías ideológicas hasta el 7, además del 5.3\% de los votos de los ciudadanos ubicados en la categoría 8. Si quisiéramos explicar dicha ventaja en términos de una variable de valencia, veríamos que sería equivalente a una diferencia personalista en favor de Dilma Roussef equivalente a 4.61 puntos del espacio ideológico en comparación a José Serra; es decir, sería el resultado esperable en términos ideológicos si José Serra se situara 4.61 puntos más a la derecha de la ubicación del PSDB, lo que incluso lo sacaría de la escala que utilizamos.

Este análisis nos diría que el efecto de Dilma Rousseff en las elecciones presidenciales de 2010 fue avasallador, obteniendo un resultado muy por encima del esperado por las posiciones ideológicas de los partidos y la distribución de la población.

Por último, examinamos los resultados de la última contienda presidencial, de 2014, para lo cual partimos de la distribución ideológica de la población brasileña para dicho año.

Tabla 9. Distribución ideológica en Brasil (2014)

\begin{tabular}{|c|c|c|c|c|c|}
\hline Nivel & Nivel & Frecuencia & Frecuencia & Acumulado & Acumulado \\
discreto & continuo & absoluta & relativa & & inverso \\
\hline 1 & $1-1.5$ & 165 & 0,1308 & 0,1308 & 1,0000 \\
\hline 2 & $1.5-2.5$ & 51 & 0,0404 & 0,1713 & 0,8692 \\
\hline 3 & $2.5-3.5$ & 95 & 0,0753 & 0,2466 & 0,8287 \\
\hline 4 & $3.5-4.5$ & 96 & 0,0761 & 0,3228 & 0,7534 \\
\hline 5 & $4.5-5.5$ & 362 & 0,2871 & 0,6098 & 0,6772 \\
\hline 6 & $5.5-6.5$ & 105 & 0,0833 & 0,6931 & 0,3902 \\
\hline 7 & $6.5-7.5$ & 82 & 0,0650 & 0,7581 & 0,3069 \\
\hline 8 & $7.5-8.5$ & 108 & 0,0856 & 0,8438 & 0,2419 \\
\hline 9 & $8.5-9.5$ & 56 & 0,0444 & 0,8882 & 0,1562 \\
\hline 10 & $9.5-10.0$ & 141 & 0,1118 & 1,0000 & 0,1118 \\
\hline
\end{tabular}

Elaboración propia con base en LAPOP.

En este caso, los datos disponibles de distribución ideológica de la población ofrecen un panorama ligeramente distinto en función de que la escala empleada varía de la $[0,10]$ en la 
que están representados los datos de 2006 y 2010 a una escala [1,10]. Ello hace que el poder focal del valor central 5 , se convierta en un valor relativamente más cercano al de la extrema izquierda. A pesar de dicho cambio de escala, es innegable que el mediano de la población se sitúa en un valor más centrado, 5 en términos de la distribución discreta, que con respecto a los datos de 2010. Sin embargo, hemos de recordar que este valor estaría situado en este caso en el centro-izquierda de la distribución.

Una vez mencionada la traslación ideológica hacia la izquierda de la población brasileña, la distribución muestra características similares a las de los años anteriores. Por ejemplo, se observan fuertes concentraciones en los dos valores extremos, y una mayor tendencia hacia el centro en el segmento centro-izquierda en comparación a la situación en el centroderecha.

Tabla 10. Resultados esperados y efectivos para las elecciones presidenciales en Brasil (2014)

\begin{tabular}{|c|c|c|c|c|}
\hline Partido & $\begin{array}{c}\text { Posición } \\
\text { partido }\end{array}$ & Plataforma partido & $\begin{array}{c}\text { Resultado esperado por } \\
\text { plataforma }\end{array}$ & Resultado final \\
\hline PT & 5.35 & $1-5.645$ & $62.19 \%$ & $51.64 \%$ \\
\hline PSDB & 5.94 & $5.645-10$ & $37.81 \%$ & $48.36 \%$ \\
\hline
\end{tabular}

Elaboración propia con base en LAPOP.

Las posiciones ideológicas en las que los partidos se situaron en 2014, muestran todavía un mayor acercamiento al centro, en comparación a los datos de 2010. En función de sus posiciones relativas y con base en el supuesto de preferencias espaciales simétricas, el punto de quiebre estaría situado en el 5.645. Ello implicaría que, en función solamente de las preferencias ideológicas el PT debería haber capturado la totalidad de los votos de los ciudadanos situados en el punto 5 y hacia la izquierda del mismo, además del 14.5\% de los votos de los ciudadanos ubicados en el punto 6. Ello le daría al PT una fuerte victoria esperada con el $62.19 \%$ de los votos.

La diferencia con el resultado final, en el que la candidata del PT, la presidenta Dilma Rousseff se hizo con la victoria, pero con un resultado notoriamente inferior, del $51.64 \%$ de los votos, reflejaría una notoria desventaja relativa en términos de la valencia de los candidatos. Para reflejar dicho resultado en función de la distribución ideológica de la población brasileña en 2014, la plataforma capturada del PT se tendría que haber limitado a [1, 5.175]. Para obtener dicho resultado, la diferencia relativa entre la candidata Dilma Rousseff, del PT, y Aécio Neves, del PSDB, tuvo que ser de 0.941 puntos en favor del segundo, medidos en la escala ideológica. Es decir, este hubiera sido el resultado esperable si el PT se hubiera situado 0.941 puntos más hacia la izquierda.

En función de la escala ideológica disponible, la ajustada victoria del PT se habría dado por cuestiones ajenas a la distribución ideológica de la población, las cuales afectaron positivamente a la oposición. Sin embargo, en función del enorme peso del punto focal 5 que se convierte en el mediano de la población, pero que no es propiamente el centro del espacio ideológico, la variable personalista podría no haber jugado un papel tan importante. 


\section{CONCLUSIONES}

La polarización bipartidista entre el PT y el PSDB ha protagonizado las segundas vueltas de las elecciones presidenciales de los últimos años en Brasil. La competencia protagonizada por estos dos partidos, a pesar de su diferenciación ideológica, no se limita a dicha dimensión. La particularidad de cada candidato puede ofrecer una dimensión añadida que también tengan en cuenta los votantes.

La observación de la distribución ideológica de los ciudadanos brasileños, su desarrollo y comparación con los resultados de las respectivas segundas vueltas de las elecciones presidenciales de Brasil, puede resultar un escenario ideal para dimensionar el efecto esperado del voto ideológico y del personalista, bajo supuestos de simetría ideológica espacial.

Este análisis indica que la victoria presidencial de Lula da Silva en 2006 no se debió a una ventaja personalista, sino a la relativa centralidad del PT, con respecto al PSDB, en materia ideológica. Dicha ventaja ideológica del PT disminuyó en 2010, con un desplazamiento a la derecha de la población y un planteamiento más centralizado del PSDB. Por ello, la victoria de Dilma Rousseff en 2010 tiene un alto componente personalista. Por último, los resultados de 2014, junto con un traslado hacia la izquierda de la ciudadanía brasileña, indican que la victoria del PT, pudo haberse debido de nuevo a razones ideológicas.

FUENTES

BIBLIOGRÁFICAS

ALCANTARA, M., Sistemas políticos de América Latina, Volumen I, América del Sur, Madrid, Editorial TECNOS, 1999.

ANSOLABEHERE, S. \& SNYDER, J., Valence politics, Public Choice, Vol. 103, 327-226, 1999.

ARAGONES, E. \& PALFREY, T., Mixed equilibrium in a Downsian Model with a Favored Candidate, Journal of Economic Theory, Vol. 103, 131-161, 2003.

DOWNS, A., An economic theory of democracy, Nueva York, Harper y Row, 1957.

HOTELLING, H. Stability in competition, Economic Journal, 39: 41-57, 1929.

HAZAN, Reuven, Centre paties: Polarization and competition in european parlamentary democracies, Londres, Editorial Pinter, 1997.

LEÓN GANATIOS, L., La teoría espacial del voto: una propuesta de aplicación en Latinoamérica, Revista Mexicana de Análisis Político y Administración Pública, 2: 57-79, 2013.

LAPOP (latinamerican public opinión project) http://datasets.americasbarometer.org/database-login/usersearch.php [visitado el 30 de noviembre de 2014]

MENEGUELLO, R., Las elecciones brasileñas de 2010: política nacional, fragmentación partidista y coaliciones. En Alcántara y Tagina (coord.) Elecciones y política en América Latina. México, Editorial Porrúa., 2013.

NOHLEN, D. Sistemas electorales y partidos políticos. México, Fondo de Cultura Económica, 2004. 
OÑATE, P. \& OCAÑA, F., Análisis de datos electorales, Madrid, Centro de Investigaciones Sociológicas, 1999.

POLITICAL DATABASE OF AMERICAS. Elecciones de 1989 en internet http://pdba.georgetown.edu/Elecdata/Brazil/pres89.html [visitado el 14 de diciembre de 2014]

Elecciones 1994 en internet http://pdba.georgetown.edu/Elecdata/Brazil/ pres94.html [visitado el 14 de diciembre de 2014]

,Elecciones 1998 en internet http://pdba.georgetown.edu/Elecdata/Brazil/ pres98.html [visitado el 14 de diciembre de 2014]

, Elecciones 2002 en internet http://pdba.georgetown.edu/Elecdata/Brazil/preso2.html [visitado el 14 de diciembre de 2014]

, Elecciones 2006 en internet http://pdba.georgetown.edu/Elecdata/Brazil/ preso6.html [visitado el 14 de diciembre de 2014]

Elecciones 2010 en internet http://pdba.georgetown.edu/Elecdata/Brazil/ pres10_1.html [visitado el 14 de diciembre de 2014]

TRIBUNAL SUPREMO ELECTORAL DE BRASIL, Elecciones 2014 en internet http://divulga.tse.jus.br/oficial/index.html. [visitado el 14 de diciembre de 2014]

SARTORI, G., Partidos y sistemas de partido, Madrid: Alianza Editorial, 1987. Partidos y sistemas de partido, Madrid: Alianza Editorial, 2009.

STOKES, D., Spatial models of party competition, American Political Science Review, Vol. 57, Núm. 2, 368-377, 1963.

WITTMAN, D.(2007) Candidate quality, pressure group endorsements and the nature of political advertising, European Journal of Political Economy, Vol. 23, 360-378, 2007. 
